\title{
An fMRI Study of Syntactic Adaptation
}

\author{
U. Noppeney and C. J. Price
}

\begin{abstract}
a

It is easier to produce and comprehend a series of sentences when they have similar syntactic structures. This "syntactic priming" effect was investigated during silent sentence reading using (i) blood oxygenation level-dependent (BOLD) response as a physiological measure in an f MRI study and (ii) reading time as a behavioral measure in a complementary self-
\end{abstract}

\section{INTRODUCTION}

Syntax determines the propositional content of a sentence by relating the meanings of the individual words to each other. Although human language is known for its vast combinatorial properties, in everyday conversations people show a tendency to employ identical syntactic structures in successive sentences. The facilitation of sentence processing that occurs when a sentence has the same syntactic form as a preceding sentence is known as "syntactic priming." Critically, this effect is seen even when lexical, metrical, and thematic features change. It has therefore been attributed to a residual activation of syntactic processes (Bock \& Griffin, 2000) or representations (Pickering, Branigan, Cleland, \& Stewart, 2000) that reduces the processing load when a syntactically similar sentence follows in close succession.

Previous behavioral studies have shown syntactic priming effects during both sentence production (Smith \& Wheeldon, 2001; Hartsuiker \& Kolk, 1998; Hartsuiker, Kolk, \& Huiskamp, 1999; Hartsuiker \& Westenberg, 2000; Pickering \& Branigan, 1998, 1999; Bock, 1986; Bock \& Griffin, 2000) and comprehension (Potter \& Lombardi, 1998; Branigan, Pickering, Liversedge, Stewart, \& Urbach, 1995; Branigan, Pickering, \& Cleland, 2000; Frazier, Tapt, Roeper, \& Clifton, 1984; Mehler \& Carey, 1967). In controlled sentence production tasks (sentence completion, picture description, sentence recall), subjects tend to convey their messages in identical syntactic forms. In sentence comprehension tasks, evidence for syntactic priming has been less consistent. While Frazier et al. (1984) reported shorter reading times for a sequence of sentences with similar syntactic

University College London paced reading paradigm. We found that reading time and left anterior temporal activation were decreased when subjects read sentences with similar relative to dissimilar syntactic forms. Thus, syntactic adaptation during sentence comprehension is demonstrated in a neural area that has previously been linked to both lexical semantic and sentence processing.

forms, Branigan et al. (1995) found a reaction time effect only for sentences with local syntactic ambiguities. One explanation for these inconsistent results is that reading time, as an end of phrase measurement, does not sensitively reflect all cognitive processes involved in sentence comprehension. However, the effect of syntactic priming on sentence comprehension can be seen using alternative measures. For instance, it has been shown that the interpretation of sentences presented in white noise is affected by the syntactic structure of the preceding sentence (Mehler \& Carey, 1967). Furthermore, evidence has been provided for comprehensionto-production priming (Pickering et al., 2000): Reading a sentence with a particular syntactic form made subjects more likely to produce a sentence with an identical syntactic form (Branigan et al., 2000). It therefore appears that syntactic aspects of prior context can influence sentence comprehension as well as production.

Although no previous neuroimaging experiment (to our knowledge) has investigated the neural correlates of syntactic priming at a production or comprehension level, the neural systems underlying sentence comprehension are well established. PET and fMRI studies have shown that sentence comprehension, relative to consonant strings (Vandenberghe, Nobre, \& Price, 2002; St George, Kutas, Martinez, \& Sereno, 1999), unrelated words (Stowe et al., 1998, 1999; Bottini et al., 1994; Mazoyer et al., 1993), environmental sounds (Humphries, Willard, Buchsbaum, \& Hickok, 2001), or rest (Chee, Tan, \& Thiel, 1999; Bavelier et al., 1997) is subserved by a widespread left-lateralized frontotemporal network extending into the temporal poles, the hippocampus, and the temporo-parieto-occipital junction. Left frontal activation has been reported with increases in (i) syntactic complexity (Keller, Carpenter, \& Just, 2001; Caplan, Alpert, \& Waters, 1998, 1999; 
Caplan, Alpert, Waters, \& Olivieri, 2000; Just, Carpenter, Keller, Eddy, \& Thulborn, 1996; Stromswold, Caplan, Alpert, \& Rauch, 1996), (ii) syntactic violations (Embick, Marantz, Miyashita, O'Neil, \& Sakai, 2000) (Moro et al., 2001; Kuperberg et al., 2000; Ni et al., 2000; Kang, Constable, Gore, \& Avrutin, 1999), and (iii) implicitly induced syntactic analysis (Dapretto \& Bookheimer, 1999). In contrast, frontal activation has not (or only at a very low threshold) been detected when the syntactic task does not involve complex syntactic analysis (Friederici, Meyer, \& von Cramon, 2000; Kuperberg et al., 2000; Meyer, Friederici, \& von Cramon, 2000; Schlosser, Aoyagi, Fulbright, Gore, \& McCarthy, 1998).

In the present study, we use both physiological and behavioral measures to investigate syntactic priming during silent sentence reading. Our physiological measure was the blood oxygenation level-dependent (BOLD) signal in fMRI that reflects the hemodynamic response to neural activity. Critically, fMRI can provide insight into cognitive processes, even when there are no overt behavioral manifestations and when no motor response is required.

To optimize the sensitivity of our paradigm to syntactic adaptation effects, subjects silently read blocks of written sentences with either similar or dissimilar syntactic structure (see Table 1). With respect to the neural locus of syntactic adaptation, our predictions are based on previous neuroimaging (PET and fMRI) studies of syntactic processing. In Vandenberghe et al. (2002), the most significant difference between reading sentences and agrammatical scrambled sentences was located in a left anterior temporal area. Since our task also involved silent reading, we predicted that blocks of

Table 1. Examples of Sentences with the Four Different Syntactic Forms

1. Clause boundary ambiguity

a) Late closure (preferred interpretation)

Example: Before the director left the $\underline{s t a g e}_{(,)}$the play began.

b) Early closure (nonpreferred interpretation)

Example: After the headmaster had left (,) the school deteriorated rapidly.

2. Reduced relative/main clause ambiguity

a) Simple active (preferred interpretation)

Example: The artist left his sculptures to the British Museum.

b) Reduced relative (nonpreferred interpretation)

Example: The child (,) left by his parents (,) played table football.

The underlined words indicate when the reader encounters the temporary syntactic ambiguity where the "preferred" as well as the "nonpreferred" syntactic forms can be assigned. sentences with syntactically similar relative to syntactically dissimilar structures would decrease activation in this left anterior temporal area. Nevertheless, we (i) identified the whole sentence reading system relative to a low-level baseline (viewing the same sentences after the letters had been converted to meaningless false fonts) and (ii) within these regions looked for differences between sentences with similar and dissimilar syntactic structures.

\section{RESULTS \\ Behavioral Study}

Sentences were read in a self-paced fashion with a key press initiating the presentation of the next sentence. Reading times were analyzed in a four-way ANOVA with the factors (i) syntactic priming (dissimilar vs. similar sentence structures), (ii) syntactic ambiguity (presence vs. absence of a comma), (iii) interpretation (preferred vs. nonpreferred), and (iv) structure (clause boundary vs. reduced relative/main clause ambiguity. This revealed a main effect of priming, $F(1,10)=4.7$, $p=.05$, ambiguity, $F(1,10)=15.4, p<.01$, structure, $F(1,10)=7.2, p<.05$, and an interaction of interpretation with priming, $F(1,10)=6.6, p<.05$, of interpretation with ambiguity, $F(1,10)=14.4, p<.01$, and of ambiguity with structure, $F(1,10)=5.2, p<$ .05 . In particular, the reading time was significantly shorter for blocks of similar (primed) than for blocks of dissimilar (unprimed) sentences, thereby illustrating a syntactic priming effect at the behavioral level. Moreover, the ambiguity effect (i.e., increased reading times in the absence of the comma) was increased for sentences with the nonpreferred interpretation indicating that subjects processed the sentences in a normal way. Table 2 shows the reaction time data collapsed over interpretation and structure.

\section{Functional Imaging Study}

Sentence reading $>$ viewing false fonts (see Figure 1, Table 3). Sentence reading relative to false fonts resulted in predominantly left-lateralized activation in the left superior temporal sulcus, middle temporal gyri spreading into the temporal poles bilaterally. Activation was also observed in the left inferior frontal gyrus/lateral fissure and the right cerebellum.

Unprimed $>$ primed sentences. The left temporal pole (coordinates: $-42,3,-27 ; z=5.8 ; p<.001$ corrected) was the only area to show significantly more activation for blocks of sentences with dissimilar relative to similar syntactic structures, see left side of Figure 1 and Table 3. To finesse our analysis, we created a further second-level model that modeled each sentence type separately. A conjunction analysis over the independent priming 
Table 2. Self-paced Reading Times

With Comma (msec)

Without Comma (msec)

\begin{tabular}{lll}
\hline Primed & $2040(133)$ & $2275(118)$ \\
Unprimed & $2098(139)$ & $2365(114)$ \\
\hline
\end{tabular}

Values are across-volunteer means $(S E)$.

effects in all sentence types and the sentence effect confirmed that priming in the left anterior temporal area occurred irrespective of sentence type (coordinates: $-42,3,-27 ; z=4.69 ; p=.08$ corrected). The priming effect for each sentence type is illustrated on the right side of Figure 1.

Furthermore, when two subjects were excluded from the fMRI analysis (because their discrimination scores or difference in recognition performance between primed and unprimed sentences was outside the group range; Subjects 1 and 9, see Appendix A, the priming effect in the left temporal pole was still significant $(p<$ .01 corrected for multiple comparisons).

Ambiguity effect and interaction between ambiguity and priming. Nonambiguous relative to ambiguous sentences was associated with increased activation in the left entorhinal cortex, possibly reflecting increased encoding processes during nonambiguous normal sentences. An enhanced priming effect for ambiguous relative to nonambiguous sentences was observed in the left basal ganglia that have previously been associated with processing of syntactic violations (see Table 3 , Friederici et al., 2000; Moro et al., 2001).

\section{Recognition Memory Tests}

After the self-paced behavioral and fMRI experiments, subjects performed a recognition memory test to compare recognition of primed relative to unprimed sentences (see Methods and Tables 4 and 5). Accuracy of confident and nonconfident recognition was indexed by the discrimination measure $\operatorname{Pr}$ (probability hit - probability false alarm; Snodgrass \& Corwin, 1988). For subjects that performed the self-paced behavioral study, the discrimination measure was significantly greater than zero. $\operatorname{Pr}=0.53, t(11)=9.1, p<.001$, two-tailed, for confident hits but not nonconfident hits $(\operatorname{Pr}=0.066)$; and the difference in recognition performance on confident hits for primed relative to unprimed sentences was not significant. Likewise, for subjects that performed the fMRI study, the discrimination rate was significantly greater than zero (limited to the new sentences that basically conformed to syntactic structures used in the fMRI study: $\operatorname{Pr}=0.47 ; t(8)=6.0, p<.001$, two-tailed) with no significant difference between recognition performance for primed and unprimed sentences, difference $=.04$, $t(8)=0.7, p>.05$, two tailed. These results suggest that

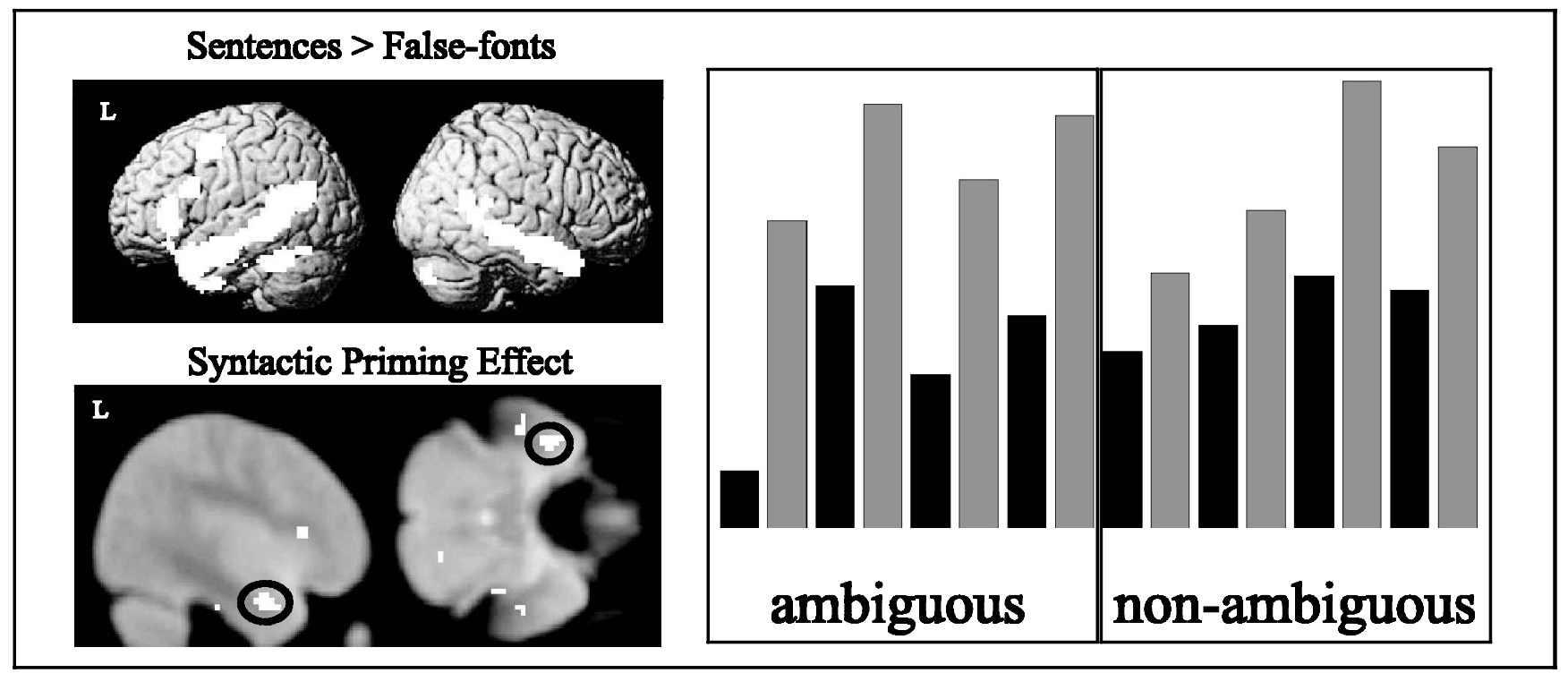

Figure 1. Top left: activation for sentences $>$ false fonts (at $p<.001$ uncorrected, spatial extent threshold $>50$ voxels for illustration purposes) rendered on the surface of the left and right hemisphere. Bottom left: activation for conjunction of sentences $>$ false fonts and unprimed $>$ primed (at $p<.001$ uncorrected, spatial extent threshold $>0$ voxels for illustration purposes) illustrating the left temporal pole activation on sagittal and axial slices on the EPI image averaged across the 12 subjects. Right: parameter estimates of each sentence type relative to baseline. For each sentence type the effect size for the primed (black) is smaller than for the unprimed (gray) sentences. From left to right: (i) no comma, preferred interpretation, reduced relative/main clause ambiguity, (ii) no comma, preferred interpretation, clause boundary ambiguity, (iii) no comma, nonpreferred interpretation, reduced relative/main clause ambiguity, (iv) no comma, nonpreferred interpretation, clause boundary ambiguity, (v) comma, preferred interpretation, reduced relative/main clause ambiguity, (vi) comma, preferred interpretation, clause boundary ambiguity, (vii) comma, nonpreferred interpretation, reduced relative/main clause ambiguity, (viii) comma, nonpreferred interpretation, clause boundary ambiguity 
Table 3. Lateral fissure

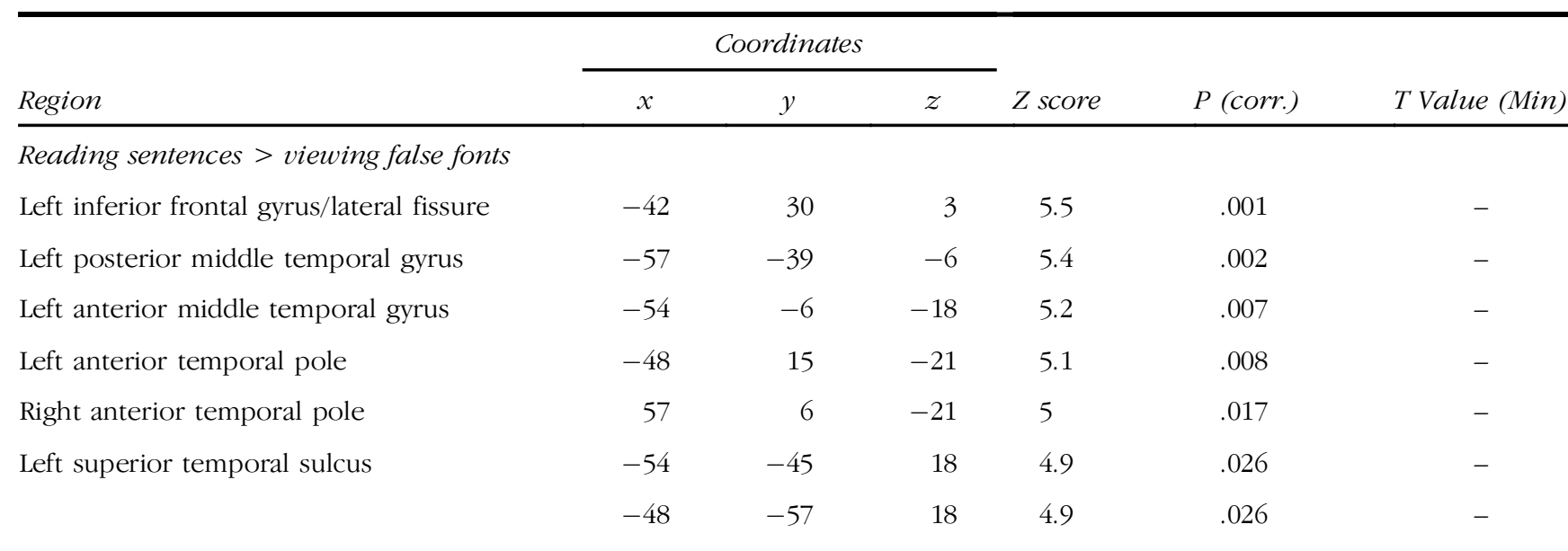

\section{Conjunction}

Unprimed $>$ primed sentences and reading sentences $>$ viewing false fonts

Left temporal pole

$\begin{array}{lll}-42 & 3 & -27\end{array}$

5.8

0

4.7

Nonambiguous $>$ ambiguous and reading sentences $>$ viewing false fonts

Left entorhinal cortex

$\begin{array}{lll}-15 & -3 & -27\end{array}$

5.07

.012

3.84

Priming effect for ambiguous > nonambiguous sentences and reading sentences $>$ viewing false fonts

Left basal ganglia

$-24$

0

12

5.12

.009

3.9

Sentences $>$ false fonts at $p<.05$ corrected; spatial extent threshold $>3$ voxels.

Unprimed $>$ primed, nonambiguous $>$ ambiguous, priming effect for ambiguous $>$ nonambiguous sentences at $p<.05$ corrected; spatial extent threshold > 1 voxel.

Coordinates according to Talairach and Tournoux. BA = Brodmann's area; $P$ (corr.) $=$ probability corrected for multiple comparisons in the entire brain; $T$ value $(\min )=$ the minimal $t$ value for each contrast.

subjects had encoded the meanings of both primed and unprimed sentences to a similar level.

\section{DISCUSSION}

The current study aimed to investigate the neural correlates of syntactic priming during silent sentence reading. First, we identified the neural system that underlies sentence comprehension relative to a low-level baseline (strings of false fonts) and, within this system, we identified the effect of syntactic priming by comparing blocks of sentences with dissimilar syntactic structures to those with similar syntactic structures.

\section{Sentence Comprehension System}

Reading sentences relative to viewing false fonts elicited widespread predominantly left-lateralized activation in the middle and inferior temporal gyri extending into the temporal poles and the hippocampus. In addition, activation was observed in the left inferior and middle frontal gyri. This pattern of activation corresponds to that previously reported during explicit sentence comprehension paradigms (Keller et al., 2001;
Chee et al., 1999; Dapretto \& Bookheimer, 1999; Bottini et al., 1994) and confirms that despite our silent reading paradigm, subjects were processing words at the syntactic and semantic level. While frontal and posterior temporo-parietal activation is commonly associated with sentence comprehension, anterior temporal activation has been reported by fewer studies (Vandenberghe et al., 2002; Humphries et al., 2001; Keller et al., 2001; Chee et al., 1999; Dapretto \& Bookheimer, 1999; St George et al., 1999; Stowe et al., 1999; Bavelier et al., 1997; Bottini et al., 1994; Mazoyer et al., 1993). The inconsistent effects in the anterior temporal cortex can be explained by several factors: First, several studies have used an experimental paradigm that did not include a low-level control condition to enable the entire sentence comprehension network to be revealed (e.g., Caplan et al., 1998; Caplan \& Waters, 1999; Caplan et al., 2000; Stromswold et al., 1996). Second, other studies have focused on regions of interest that did not include the inferior anterior temporal regions (Embick et al., 2000; Kuperberg et al., 2000; Ni et al., 2000; Carpenter, Just, Keller, Eddy, \& Thulborn, 1999; Kang et al., 1999; Just et al., 1996). Finally, BOLD signal within the temporal poles might 
Table 4. Recognition Performance for New and Old Sentences (Self-paced Reading Study)

Recognition Judgment

\begin{tabular}{lllll}
\cline { 3 - 4 } Word Type & Sure Old & Unsure Old & Sure New & Unsure New \\
\hline Proportion of responses & & & & \\
Old & $0.61(0.22)$ & $0.1(0.09)$ & $0.19(0.22)$ & $0.09(0.07)$ \\
$\quad$ Primed & $0.63(0.22)$ & $0.09(0.09)$ & $0.19(0.23)$ & $0.09(0.10)$ \\
$\quad$ Unprimed & $0.59(0.22)$ & $0.12(0.09)$ & $0.2(0.22)$ & $0.09(0.05)$ \\
New & $0.08(0.03)$ & $0.09(0.08)$ & $0.58(0.27)$ & $0.24(0.23)$ \\
\hline
\end{tabular}

Values are across-volunteer means $(S D)$.

have been missed due to fMRI susceptibility artefacts (Lipschutz, Friston, Ashburner, Turner, \& Price, 2001; Devlin et al., 2000).

Evidence for a widespread sentence comprehension system encompassing temporal as well as frontal brain regions has also been provided by recent neuropsychological studies (Caplan, Baker, \& Dehaut, 1985; Caplan, Hildebrandt, \& Makris, 1996). To our knowledge the most comprehensive study (Caplan et al., 1996) that investigated the association between lesion localization and sentence comprehension deficits has demonstrated that patients with only posterior lesions were as impaired as those with anterior and posterior lesions on a variety of syntactic operations. Other neuropsychological studies have argued against the general importance of Broca's area for sentence comprehension and have limited its role to specific types of syntactic operations. For instance, Grodzinsky (1995) and Grodzinsky (2000) have investigated sentence comprehension in agrammatic Broca's aphasics and hypothesized that the left inferior frontal gyrus is involved only in the computation of the relation between transformationally moved phrasal constituents (but see Berndt, Mitchum, \& Haendiges, 1996; Berndt \& Carama, 1999; Caplan, 1995, for controversial discussion of method and results). Based

Table 5. Recognition Performance for New and Old Sentences (fMRI Study)

\begin{tabular}{lcc}
\hline \multirow{2}{*}{ Word Type } & \multicolumn{2}{c}{ Recognition Judgment } \\
\cline { 2 - 3 } Proportion of Responses & \multicolumn{1}{c}{ Old } & New \\
Old & $0.83(0.1)$ & $0.17(0.1)$ \\
$\quad$ Primed & $0.84(0.2)$ & $0.16(0.2)$ \\
$\quad$ Unprimed & $0.80(0.2)$ & $0.20(0.2)$ \\
New & $0.35(0.2)$ & $0.65(0.2)$ \\
\hline
\end{tabular}

Values are across-volunteer means $(S D)$.

Results restricted to the new sentences with syntactic structures as in fMRI study. on reports that lesions in Broca's area failed to cause sentence comprehension deficits, Dronkers, Wilkins, Van Valin, Redfern, and Jaeger (1994) investigated whether lesions in other brain regions were necessarily associated with morphosyntactic comprehension disorders. They argued for a specific role of the anterior portion of BA22, which was commonly lesioned in aphasics with poor morphosyntactic comprehension while being spared in aphasics with good sentence comprehension (Dronkers et al., 1994; Dronkers, Redfern, \& Knight, 2000). In line with this emphasis on the functional importance of temporal brain regions, patients with anterior medial and lateral temporal lobe damage subsequent to herpes encephalitis have also been shown to be impaired in comprehending ambiguous sentences relative to patients with damage restricted to the hippocampal formation (Schmolck, Stefanacci, \& Squire, 2000).

Consistent with our functional imaging results, these neuropsychological findings seem to suggest that sentence comprehension is subserved by a widespread system of anterior/posterior temporal regions in addition to frontal involvement. Further research, however, is needed to investigate the specific contributions and operations of different brain regions and potential compensatory strategies after lesions within the system in order to explain the variable neuropsychological findings.

\section{Syntactic Priming Effect}

Most importantly, we found that the syntactic priming effect was reflected behaviorally by decreased reading times, and physiologically by attenuated responses in the left temporal pole. These syntactic priming effects are unlikely to reflect differences in the following:

1. Executive demands, because sentences were read without an additional behavioral task.

2. Sentence complexity, because exactly the same sentences were presented in each condition across subjects.

3. Visual input because in the fMRI study, word presentation was held constant. 
4. Lexical semantic/repetition priming, because these factors were controlled across conditions. Furthermore, lexical repetition priming has been shown to reduce activation in the left inferior frontal and the left posterior inferior temporal gyri (Buckner, Koutstaal, Schacter, \& Rosen, 2000; Wagner, Koutstaal, Maril, Schacter, \& Buckner, 2000; Schacter \& Buckner, 1998).

We therefore demonstrate that reading time and anterior temporal activation are reduced when consecutive sentences are syntactically similar. As the behavioral and the fMRI study used slightly different experimental designs and stimulus presentation modes (cf. Methods for further information), we cannot clearly link the syntactic priming effect at the behavioral level with the syntactic adaptation effect at the neural level. Thus, for instance, one might hypothesize that the serial presentation mode decreases the depth of syntactic processing. However, serial presentation is commonly used in EEG studies of sentence reading, indicating that it does not prevent subjects from processing a series of words at the syntactic level. Moreover, one EEG study demonstrated a reduced $\mathrm{P} 600$ effect for processing syntactically primed sentences (Branigan et al., 1995). Finally, even in the absence of a behavioral syntactic priming effect, f MRI might still be able to demonstrate a neural syntactic adaptation effect because fMRI is sensitive to changes in different cognitive processes involved during sentence processing, which might be missed by evaluating sentence reading time as an end of phrase measure. In summary, our study (i) provides further evidence that syntactic aspects of prior context (at least for the four types of syntactic structures used in our experiment) can influence and facilitate sentence comprehension and (ii) demonstrates that this effect might be expressed as an attenuation of neural activity in the left anterior temporal lobe.

Previous behavioral studies have not only provided evidence for comprehension-comprehension priming (as in our study) but have also demonstrated that priming occurs between comprehension and production (i.e., intermodal comprehension-production priming). Based on this common effect, Branigan et al. (1995) (though see Bock \& Griffin, 2000) hypothesized that priming results from persistent activation of syntactic representations that are accessed during production as well as comprehension. For instance, in our study (see Table 1), prior presentation of early/late closure sentences, would result in priming of transitive/intransitive subcategorization frames: Thus, an "early-closure" sentence primes the intransitive interpretation of the verb in the target sentence and predisposes the subsequent noun as the subject of the next phrase. Within this theoretical framework, the deactivation in the left anterior temporal lobe for syntactically primed sentences may reflect the persistence of syntactic representations, which facilitate the combination of individual word meanings into a semantic representation of the entire sentence. Alternatively, as syntactic forms might be linked to particular thematic representations, subjects might rely on thematic templates resulting in decreased demands on syntactic processing. Finally, the decreased temporal pole activation might reflect decreased attentional demands and computational effort during reading of syntactically primed sentences. Further research is clearly needed to disentangle and specify the contributions of lexical/sentential semantics and syntactic frames in the process of semantic-syntactic integration. Moreover, contrary to Branigan et al.'s hypothesis, syntactic priming might be a complex phenomenon affecting different processes/representations associated with several brain regions, so that future research is needed to extend these findings to other types of syntactic structures and to investigate the neural basis of syntactic priming in sentence production.

Finally, we will discuss the relationship between the anterior temporal region and semantic-syntactic integration within the context of previous functional imaging results. The anterior temporal region (coordinates: $-42,3,-27$ ) we found to be modulated by syntactic priming corresponds to the area that has previously been associated with (i) lexical semantic priming (coordinates: $-40,4,-28$; Rossell, Price, \& Nobre, 2003; Mummery, Shallice, \& Price, 1999) and (ii) lexical semantic processing in general (e.g., coordinates: $-42,0$, -28; Price, Moore, Humphreys, \& Wise, 1997; Vandenberghe, Price, Wise, Josephs, \& Frackowiak, 1996). (iii) Temporal pole activation has also been reported for sentences relative to unrelated words (Stowe et al., 1999; Bottini et al., 1994; Mazoyer et al., 1993) or environmental sounds (Humphries et al., 2001). In particular, it has been shown for grammatical structure irrespective of whether the semantic content was pragmatically correct (coordinates: $-44,-6,-24$; Vandenberghe et al., 2002). (iv) Left anterior temporal pole activation is also enhanced for stories relative to unrelated sentences (Fletcher et al., 1995). These results indicate that the anterior temporal pole plays an important role in cognitive processes that involve semantic integration at the lexical, sentential and discourse level.

\section{Conclusion}

In conclusion, our behavioral and functional imaging data suggest that if successive sentences follow the same syntactic structure, it is less demanding for the reader to assign thematic roles to the sentence arguments. Essentially, it becomes easier to determine "who does what to whom" in the sentence. This effect is reflected by attenuated neural activity in an anterior temporal area previously linked to both semantic and syntactic processing. 


\section{METHODS}

\section{Subjects}

After giving informed consent, 25 healthy, native English speakers participated in the study: 12 in the fMRI experiment ( 9 men, mean age 24) and 13 in the behavioral experiment. Two subjects were excluded from the analysis of the behavioral experiment because their reading times were three standard deviations above the group's mean (remaining 11 subjects: 3 men, mean age 24). Three of the subjects in the fMRI experiment did not perform the postscan recognition test.

\section{Design}

Two experiments (one behavioral and one fMRI) were conducted on two different groups of subjects. In each experiment, subjects silently read blocks of nine-word sentences with four possible syntactic forms (see Table 1 for details). Within a block, there were five sentences that were either all of the same type (syntactically similar structures) or a mixture of all four syntactic types (syntactically dissimilar structures). Care was taken that the repetition of content words and semantic relatedness of subsequent sentences within a block were controlled across primed and unprimed conditions. In addition, the degree of syntactic ambiguity was modulated creating a fully balanced $2 \times 2$ factorial design. In the less ambiguous conditions, sentences were presented with commas indicating the boundaries of the clauses. In the syntactically ambiguous conditions, sentences were presented without a comma. In this article, we will focus only on the syntactic priming effect.

\section{Stimuli}

There were 160 sentences in total with four possible syntactic forms and two types of syntactic ambiguity: "clause boundary ambiguity" and "reduced relative/main clause ambiguity." In sentences with clause boundary ambiguity, the noun after the verb can either be interpreted as the direct object of the preposed clause ("late closure," the preferred interpretation) or as the subject of the subsequent main clause ("early closure," the nonpreferred interpretation). In sentences with "reduced relative/main clause ambiguity" the verb can be interpreted as the main verb of the sentence ("simple active," the preferred interpretation) or as a "reduced relative" (the nonpreferred interpretation). Thus, the design can be described in a more detailed way as $2 \times$ $2 \times 2 \times 2$ design with the factors (i) syntactic priming (dissimilar vs. similar sentence structures), (ii) syntactic ambiguity (presence vs. absence of a comma), (iii) interpretation (preferred vs. nonpreferred) and (iv) structure (clause boundary vs. reduced relative/main clause ambiguity. The sentences (with and without commas) were rotated across conditions over subjects and therefore counterbalanced for primed versus unprimed and ambiguous versus unambiguous comparisons.

\section{Tasks}

During both experiments, subjects were asked to read the sentences silently for meaning and informed that we would monitor their eye movements to ensure they were attending to the words. There were no other instructions in the fMRI study, but in the behavioral experiment, subjects made a key press response as soon as they had read and understood each sentence. The key press then triggered the presentation of the next sentence. An explicit comprehension task was not included, as this would have induced abnormal reading strategies and increased the demands on working memory and executive functions. To ensure that the sentences were read subjects were given a surprise recognition test after the experimental data were collected (see below). In addition, (1) continuous monitoring of eye movements confirmed that all subjects were attending to the stimuli and (2) activation throughout the sentence comprehension system confirmed that subjects were processing the sentences at a syntactic and semantic level.

Blocks of sentences were interleaved with rest in the behavioral study and a baseline condition in the fMRI experiment. During the fMRI baseline, visual input was controlled as subjects attentively viewed the sentences of the pre/succeeding block after all the letters were transformed into false fonts. The rest condition in the behavioral study allowed us to replicate the fMRI procedure and ensure maximum attention during the sentence conditions.

\section{Procedure}

In the behavioral study, all nine words of a sentence were presented simultaneously on the computer screen until the key press response triggered the next sentence. In the f MRI study, sentences were presented one word at a time at a fixed pace (word duration $300 \mathrm{msec}$, SOA of words $350 \mathrm{msec}$, SOA of sentences $4350 \mathrm{msec}$, block length for five sentences $=22 \mathrm{sec}$ ). Thus, we chose different presentation modes for the two experiments: In the behavioral study, we used a simultaneous word presentation mode, because a serial presentation mode at a fixed pace would have decreased the sensitivity of reading time as an end of phrase measure. By contrast, fMRI provides insight into cognitive processes even in the absence of behavioral manifestations (cf. f MRI adaptation effects in object recognition (Grill-Spector et al., 1999; Grill-Spector \& Malach, 2001). Therefore, in f MRI we used the more stringent serial presentation mode to control for visual input and eye movements without 
removing general syntactic (e.g., Caplan et al., 2002; Vandenberghe et al., 2002) or syntactic priming effects (Branigan et al., 1995).

\section{Recognition Memory Test}

To ensure that subjects had processed the syntactic properties of the sentences during the behavioral and fMRI experiments, they were given a surprise recognition test. This occurred about $5 \mathrm{~min}$ after the completion of the behavioral experiment and $15 \mathrm{~min}$ after the completion of the fMRI experiment. The test required subjects to decide whether a sentence had been read before in the experiment (old/new judgement) by yes/no key presses (behavioral study) or by marking a response sheet (f MRI study). Subjects were only asked to be as accurate as possible; response time was not emphasized.

For the subjects in the behavioral experiment, all 160 of the "old" sentences were represented intermixed with 160 additional new sentences. In addition to making old/new judgements, these subjects also indicated whether or not they were confident about their decision. For the subjects in the fMRI study, 29 sentences randomly drawn from the 160 old sentences were represented intermixed with 23 additional new sentences (10 of the new sentences basically conformed to the syntactic structures used in the fMRI study).

\section{fMRI Scanning Technique}

A 2-T Siemens Vision system was used to acquire both $\mathrm{T} 1$ anatomical volume images and $\mathrm{T} 2 *$-weighted axial echoplanar images (gradient-echo, Cartesian $k$-space sampling, TE $=40 \mathrm{msec}$, TR $2.89 \mathrm{sec}$, slices acquired sequentially in descending direction, matrix $64 \times 64$, spatial resolution $3 \times 3 \times 3-\mathrm{mm}^{3}$ voxels, interslice gap $1.2 \mathrm{~mm}$, slice thickness $1.8 \mathrm{~mm}, 38$ slices covering nearly the whole brain) with BOLD contrast. To avoid Nyquist ghost artefacts a generalized reconstruction algorithm was used for data processing (Josephs, Deichmann, \& Turner, 2000). A total of 518 volume images were taken continuously in two sessions. The first six volumes were discarded to allow for T1 equilibration effects.

\section{Data Analysis}

Data were preprocessed using SPM99 and statistical analysis was performed using SPM2. All volumes were realigned to the first volume and resliced using a sinc interpolation and a mean realigned image was created. The mean image was used to determine the parameters applied to all volumes during spatial normalization and resampling to a standard template. All functional vol- umes were then smoothed with an 8-mm full width at half maximum isotropic Gaussian kernel. The T1-weighted structural MRI scans were coregistered to the mean fMRI image for each subject and transformed into the Talairach and Tournoux space. The time series (f MRI) in each voxel was high-pass filtered to $1 / 100 \mathrm{~Hz}$ and globally normalized with proportional scaling.

The fMRI experiment was modeled in an event related fashion with regressors entered into the design matrix after convolving each event-related stick function with a canonical hemodynamic response function. The model consisted of 16 activation conditions arranged in a $2 \times 2 \times 2 \times 2$ factorial design with the factors (i) syntactic priming, (ii) syntactic ambiguity/presence of a comma, (iii) interpretation (preferred/nonpreferred), and (iv) structure (clause boundary/reduced relative ambiguity) and one common control condition (i.e., sentences after being transformed into false fonts). Nuisance covariates included the realignment parameters (to account for motion artefacts). Condition-specific effects for each subject were estimated according to the general linear model (Friston et al., 1995) and passed to a second-level analysis as contrasts. This involved creating contrast images for each subject and three secondlevel ANOVAs that modeled

(i) the sentence effect (all sentences $>$ control condition) and

(ii) one of the following effects:
a. priming,
b. ambiguity, or
c. interaction between ambiguity and priming.

Inferences were made at the second level to emulate a random effects analysis and allow generalization to the population (Friston, Holmes, Price, Buchel, \& Worsley, 1999).

Analysis of the data tested for the following:

(I) Reading sentences $>$ viewing false fonts.

(II) Syntactic priming: unprimed > primed and vice versa.

(III) Syntactic ambiguity: ambiguous > nonambiguous and vice versa.

(IV) Interaction of ambiguity and priming: unprimed ambiguous-primed ambiguous $>$ unprimed nonambiguous-primed nonambiguous.

To limit the search space for the effects pertaining to contrasts (II), (III), (IV) to areas that were activated for sentences relative to false fonts, they were tested for in conjunction with the sentence effect. The conjunction identifies areas where activation is significant for both contrasts. We report activations at a significance threshold of $p<.05$ (corrected for multiple comparisons for the entire brain) and an extent threshold of $>1$ voxel (differential effects) and $>3$ voxels sentence main effect. 
APPENDIX

Self-paced Reading Times

\begin{tabular}{|c|c|c|c|c|}
\hline Priming & Ambiguity & Interpretation & $\begin{array}{l}\text { Syntactic } \\
\text { Structure }\end{array}$ & $\begin{array}{c}\text { ReadingTime } \\
\text { (msec) }\end{array}$ \\
\hline \multirow[t]{8}{*}{ Primed } & No comma & Preferred & $\mathrm{RR}$ & 2013 (354) \\
\hline & & & Wh & $2280(475)$ \\
\hline & & Nonpreferred & $\mathrm{RR}$ & $2352(600)$ \\
\hline & & & Wh & 2455 (398) \\
\hline & Comma & Preferred & $\mathrm{RR}$ & 1958 (428) \\
\hline & & & Wh & 2055 (386) \\
\hline & & Nonpreferred & $\mathrm{RR}$ & $2104(504)$ \\
\hline & & & Wh & $2044(567)$ \\
\hline \multirow[t]{8}{*}{ Unprimed } & No comma & Preferred & $\mathrm{RR}$ & 2196 (395) \\
\hline & & & Wh & $2351(534)$ \\
\hline & & Nonpreferred & $\mathrm{RR}$ & $2420(588)$ \\
\hline & & & Wh & $2494(290)$ \\
\hline & Comma & Preferred & $\mathrm{RR}$ & $2156(432)$ \\
\hline & & & Wh & $2160(577)$ \\
\hline & & Nonpreferred & $\mathrm{RR}$ & 2003 (461) \\
\hline & & & Wh & $2074(456)$ \\
\hline
\end{tabular}

Values are across-volunteer means $(S D)$.

$\mathrm{RR}=$ reduced relative/main clause ambiguity; $\mathrm{Wh}=$ clause boundary ambiguity.

Recognition Performance (Single-Subject Data)

\begin{tabular}{cccccc}
\hline Subjects & Correct Rejections & False Alarms & Hits & Misses & Discrimination \\
\hline 1 & 0.10 & 0.90 & 0.90 & 0.10 & 0.00 \\
2 & 0.90 & 0.10 & 0.59 & 0.41 & 0.49 \\
3 & 0.90 & 0.10 & 0.93 & 0.07 & 0.83 \\
4 & 0.70 & 0.30 & 0.90 & 0.10 & 0.60 \\
5 & 0.70 & 0.30 & 0.72 & 0.28 & 0.42 \\
6 & 0.70 & 0.30 & 1.00 & 0.00 & 0.70 \\
7 & 0.60 & 0.40 & 0.72 & 0.28 & 0.32 \\
8 & 0.50 & 0.50 & 0.97 & 0.03 & 0.47 \\
9 & 0.71 & 0.29 & 0.72 & 0.28 & 0.43 \\
\hline Mean $(S D)$ & $0.65(0.2)$ & $0.35(0.2)$ & $0.83(0.1)$ & $0.17(0.1)$ & $0.47(0.2)$ \\
\hline
\end{tabular}


Recognition Performance for Primed and Unprimed Sentences (Single-Subject Data)

\begin{tabular}{|c|c|c|c|c|c|}
\hline \multirow[t]{2}{*}{ Subjects } & \multicolumn{2}{|c|}{ Correct } & \multicolumn{2}{|c|}{ False } & \multirow{2}{*}{$\frac{\text { Difference }}{\text { Primed - Unprimea }}$} \\
\hline & Primed & Unprimed & Primed & Unprimed & \\
\hline 1 & .95 & .80 & .05 & .20 & 0.15 \\
\hline 2 & .53 & .64 & .47 & .36 & -0.11 \\
\hline 3 & .87 & 1.00 & .13 & .00 & -0.13 \\
\hline 4 & .86 & .93 & .14 & .07 & -0.08 \\
\hline 5 & .79 & .67 & .21 & .33 & 0.12 \\
\hline 6 & 1.00 & 1.00 & .00 & .00 & 0.00 \\
\hline 7 & .71 & .73 & .29 & .27 & -0.02 \\
\hline 8 & 1.00 & .93 & .00 & .07 & 0.07 \\
\hline 9 & .87 & .50 & .13 & .50 & 0.37 \\
\hline Mean $(S D)$ & $0.84(0.2)$ & $0.80(0.2)$ & $0.16(0.2)$ & $0.2(0.2)$ & $0.04(0.2)$ \\
\hline
\end{tabular}

Results limited to the new sentences with syntactic structures as in f MRI study.

\section{Acknowledgments}

This work was supported by the Wellcome Trust. We thank Oliver Josephs, Leun Otten, and Ric Henson for their helpful advice, and the radiographers at the Functional Imaging Laboratory for their assistance in collecting the data.

Reprint requests should be sent to U. Noppeney, Wellcome Department of Imaging Neuroscience, Institute of Neurology, University College London, 12 Queen Square, London WC1N 3BG, UK, or via e-mail: u.noppeney@fil.ion.ucl.ac.uk.

The data reported in this experiment have been deposited in the fMRI Data Center (http://www.fmridc.org). The accession number is 2-2004-115RC.

\section{REFERENCES}

Bavelier, D., Corina, D., Jezzard, P., Padmanabhan, S., Clark, V. P., Karni, A., Prinster, A., Braun, A., Lalwani, A., Rauschecker, J. P., Turner, R., \& Neville, H. (1997). Sentence reading: A functional MRI study at 4 Tesla. Journal of Cognitive Neuroscience, 9, 664-686.

Berndt, R. S., \& Caramazza, A. (1999). How "regular" is sentence comprehension in Broca's aphasia? It depends on how you select the patients [see comments]. Brain and Language, 67, 242-247.

Berndt, R. S., Mitchum, C. C., \& Haendiges, A. N. (1996). Comprehension of reversible sentences in "agrammatism": A meta-analysis. Cognition, 58, 289-308.

Bock, J. K. (1986). Syntactic persistence in language production. Cognitive Psychology, 18, 355-387.

Bock, K., \& Griffin, Z. M. (2000). The persistence of structural priming: Transient activation or implicit learning? Journal of Experimental Psychology. General, 129, 177-192.

Bottini, G., Corcoran, R., Sterzi, R., Paulesu, E., Schenone, P., Scarpa, P., Frackowiak, R. S., \& Frith, C. D. (1994). The role of the right hemisphere in the interpretation of figurative aspects of language. A positron emission tomography activation study. Brain, 117, 1241-1253.
Branigan, H. P., Pickering, M. J., \& Cleland, A. A. (2000). Syntactic co-ordination in dialogue. Cognition, 75, 13-25.

Branigan, H. P. Pickering, M. J., Liversedge, S. P., Stewart, A. J., \& Urbach, T. P. (1995). Syntactic priming: Investigating the mental representation of language. Journal of Psycholinguistic Research, 24, 489-506.

Buckner, R. L., Koutstaal, W., Schacter, D. L., \& Rosen, B. R. (2000). Functional MRI evidence for a role of frontal and inferior temporal cortex in amodal components of priming. Brain, 123, 620-640.

Caplan, D. (1995). Issues arising in contemporary studies of disorders of syntactic processing in sentence comprehension in agrammatic patients. Brain and Language, 50, 325-338.

Caplan, D., Alpert, N., \& Waters, G. (1998). Effects of syntactic structure and propositional number on patterns of regional cerebral blood flow. Journal of Cognitive Neuroscience, 10 , 541-552.

Caplan, D., Alpert, N., \& Waters, G. (1999). PET studies of syntactic processing with auditory sentence presentation. Neurolmage, 9, 343-351.

Caplan, D., Alpert, N., Waters, G., \& Olivieri, A. (2000). Activation of Broca's area by syntactic processing under conditions of concurrent articulation. Human Brain Mapping, 9, 65-71.

Caplan, D., Baker, C., \& Dehaut, F. (1985). Syntactic determinants of sentence comprehension in aphasia. Cognition, 21, 117-175.

Caplan, D., Hildebrandt, N., \& Makris, N. (1996). Location of lesions in stroke patients with deficits in syntactic processing in sentence comprehension. Brain, 119, 933-949.

Caplan, D., Vijayan, S., Kuperberg, G., West, C., Waters, G., Greve, D., \& Dale, A. M. (2002). Vascular responses to syntactic processing: Event-related fMRI study of relative clauses. Human Brain Mapping, 15, 26-38.

Caplan, D., \& Waters, G. S. (1999). Verbal working memory and sentence comprehension. Behavioral and Brain Sciences, 22, 77-94.

Carpenter, P. A., Just, M. A., Keller, T. A., Eddy, W. F., \& Thulborn, K. R. (1999). Time course of f MRI-activation in 
language and spatial networks during sentence

comprehension. Neuroimage, 10, 216-224.

Chee, M. W., Tan, E. W., \& Thiel, T. (1999). Mandarin and English single word processing studied with functional magnetic resonance imaging. Journal of Neuroscience, 19, 3050-3056.

Dapretto, M., \& Bookheimer, S. Y. (1999). Form and content: Dissociating syntax and semantics in sentence comprehension. Neuron, 24, 427-432.

Devlin, J. T., Russell, R. P., Davis, M. H., Price, C. J., Wilson, J., Moss, H. E., Matthews, P. M., \& Tyler, L. K. (2000). Susceptibility-induced loss of signal: Comparing PET and f MRI on a semantic task. Neuroimage, 11, 589-600.

Dronkers, N. F., Redfern, B. B., \& Knight, R. T. (2000). The neural architecture of language disorder. In: M. S. Gazzaniga (Ed.), The new cognitive neurosciences (pp. 949-960). Cambridge: MIT Press.

Dronkers, N. F., Wilkins, D. P., Van Valin, R. D., Redfern, B. B., \& Jaeger, J. J. (1994). A reconsideration of the brain areas involved in the disruption of morphosyntactic comprehension. Brain and Language, 74, 461-462.

Embick, D., Marantz, A., Miyashita, Y., O’Neil, W., \& Sakai, K. L. (2000). A syntactic specialization for Broca's area. Proceedings of the National Academy of Sciences, U.S.A., 97, 6150-6154.

Fletcher, P. C., Happe, F., Frith, U., Baker, S. C., Dolan, R. J., Frackowiak, R. S., \& Frith, C. D. (1995). Other minds in the brain: A functional imaging study of "theory of mind" in story comprehension. Cognition, 57, 109-128.

Frazier, L., Tapt, L., Roeper, T., \& Clifton, C. (1984). Parallel structure: A source of facilitation in sentence comprehension. Memory and Cognition, 12, 421-430.

Friederici, A. D., Meyer, M., \& von Cramon, D. Y. (2000). Auditory language comprehension: An event-related f MRI study on the processing of syntactic and lexical information. Brain and Language, 74, 289-300.

Friston, K. J., Holmes, A., Worsley, K. J., Poline, J. B., Frith, C. D., \& Frackowiak, R. (1995). Statistical parametric mapping: A general linear approach. Human Brain Mapping, 2, 189-210.

Friston, K. J., Holmes, A. P., Price, C. J., Buchel, C., \& Worsley, K. J. (1999). Multisubject f MRI studies and conjunction analyses. Neuroimage, 10, 385-396.

Grill-Spector, K., Kushnir, T., Edelman, S., Avidan, G., Itzchak, Y., \& Malach, R. (1999). Differential processing of objects under various viewing conditions in the human lateral occipital complex. Neuron, 24, 187-203.

Grill-Spector, K., \& Malach, R. (2001). f MRI-adaptation: A tool for studying the functional properties of human cortical neurons [Abstract]. Acta Psychologica, 107, 293-231.

Grodzinsky, Y. (1995). A restrictive theory of agrammatic comprehension. Brain and Language, 50, 27-51.

Grodzinsky, Y. (2000). The neurology of syntax: Language use without Broca's area. Behavioral and Brain Sciences, 23, $1-21$.

Hartsuiker, R. J., \& Kolk, H. H. (1998). Syntactic facilitation in agrammatic sentence production. Brain and Language, 62, 221-254.

Hartsuiker, R. J., Kolk, H. H. J., \& Huiskamp, P. (1999). Priming word order in sentence production. Quarterly Journal of Experimental Psychology. A, Human Experimental Psychology, 52, 129-147.

Hartsuiker, R. J., \& Westenberg, C. (2000). Word order priming in written and spoken sentence production. Cognition, 75, 27-39.

Humphries, C., Willard, K., Buchsbaum, B., \& Hickok, G. (2001). Role of anterior temporal cortex in auditory sentence comprehension: An f MRI study. NeuroReport, 12, 1749-1752.

Josephs, O., Deichmann, R., \& Turner, R. (2000). Trajectory measurement and generalized reconstruction in rectilinear EPI [Abstract]. ISMRM Meeting, 1517.

Just, M. A., Carpenter, P. A., Keller, T. A., Eddy, W. F., \& Thulborn, K. R. (1996). Brain activation modulated by sentence comprehension. Science, 274, 114-116.

Kang, A. M., Constable, R. T., Gore, J. C., \& Avrutin, S. (1999). An event-related f MRI study of implicit phrase-level syntactic and semantic processing. Neuroimage, 10, 555-561.

Keller, T. A., Carpenter, P. A., \& Just, M. A. (2001). The neural bases of sentence comprehension: A f MRI examination of syntactic and lexical processing. Cerebral Cortex, 11, 223-237.

Kuperberg, G. R., McGuire, P. K., Bullmore, E. T., Brammer, M. J., Rabe-Hesketh, S., Wright, I. C., Lythgoe, D. J., Williams, S. C., \& David, A. S. (2000). Common and distinct neural substrates for pragmatic, semantic, and syntactic processing of spoken sentences: An f MRI study. Journal of Cognitive Neuroscience, 12, 321-341.

Lipschutz, B., Friston, K. J., Ashburner, J., Turner, R., \& Price, C. J. (2001). Assessing study-specific regional variations in f MRI signal. Neuroimage, 1, 1-7.

Mazoyer, B. M., Tzourio, N., Frak, V., Syrota, A., Murayama, N., Levrier, O., Salamon, G., Dehaene, S., Chohen, L., \& Mehler, J. (1993). The cortical representation of speech. Journal of Cognitive Neuroscience, 5, 467-479.

Mehler, J., \& Carey, P. W. (1967). Role of surface and base structure in the perception of sentences. Journal of Verbal Learning and Verbal Behaviour, 6, 335-338.

Meyer, M., Friederici, A. D., \& von Cramon, D. Y. (2000). Neurocognition of auditory sentence comprehension: Event related f MRI reveals sensitivity to syntactic violations and task demands. Brain Research. Cognitive Brain Research, 9, 19-33.

Moro, A., Tettamanti, M., Perani, D., Donati, C., Cappa, S. F., \& Fazio, F. (2001). Syntax and the brain: Disentangling grammar by selective anomalies. Neuroimage, 13, $110-118$.

Mummery, C. J., Shallice, T., \& Price, C. J. (1999). Dual-process model in semantic priming: A functional imaging perspective. Neuroimage, 9, 516-525.

Ni, W., Constable, R. T., Mencl, W. E., Pugh, K. R., Fulbright, R. K., Shaywitz, S. E., Shaywitz, B. A., Gore, J. C., \& Shankweiler, D. (2000). An event-related neuroimaging study distinguishing form and content in sentence processing. Journal of Cognitive Neuroscience, 12, $120-133$.

Pickering, M. J., \& Branigan, H. P. (1998). The representation of Verbs:Evidence from syntactic priming. Journal of Memory and Language, 39, 633-651.

Pickering, M. J., \& Branigan, H. P. (1999). Syntactic priming in language production. Trends in Cognitive Sciences, 3, 136-141.

Pickering, M. J., Branigan, H. P., Cleland, A. A., \& Stewart, A. J. (2000). Activation of syntactic information during language production. Journal of Psycholinguistic Research, 29, 205-216.

Potter, M. C., \& Lombardi, L. (1998). Syntactic priming. Journal of Memory and Language, 1998, 265-282.

Price, C., Moore, C., Humphreys, G., \& Wise, R. (1997). Segregating semantic from phonological processing. Journal of Cognitive Neuroscience, 9, 727-733.

Rossell, S. L., Price, C. J., \& Nobre, A. C. (2003). The anatomy and time course of semantic priming investigated by f MRI and ERPs. Neuropsychologia, 41, $550-564$. 
Schacter, D. L., \& Buckner, R. L. (1998). Priming and the brain. Neuron, 20, 185-195.

Schlosser, M. J., Aoyagi, N., Fulbright, R. K., Gore, J. C., \& McCarthy, G. (1998). Functional MRI studies of auditory comprehension. Human Brain Mapping, 6, 1-13.

Schmolck, H., Stefanacci, L., \& Squire, L. R. (2000). Detection and explanation of sentence ambiguity are unaffected by hippocampal lesions but are impaired by larger temporal lobe lesions. Hippocampus, 10, 759-770.

Smith, M., \& Wheeldon, L. (2001). Syntactic priming in spoken sentence production-An online study. Cognition, 78, 123-164.

Snodgrass, J. G., \& Corwin, J. (1988). Pragmatics of measuring recognition memory: Applications to dementia and amnesia. Journal of Experimental Psychology, 117, 34-50.

St George, M., Kutas, M., Martinez, A., \& Sereno, M. I. (1999). Semantic integration in reading: Engagement of the right hemisphere during discourse processing. Brain, 122, 1317-1325.

Stowe, L. A., Broere, C. A., Paans, A. M., Wijers, A. A.,
Mulder, G., Vaalburg, W., \& Zwarts, F. (1998). Localizing components of a complex task: Sentence processing and working memory. NeuroReport, 9, 2995-2999.

Stowe, L. A., Paans, A. M., Wijers, A. A., Zwarts, F., Mulder, G., \& Vaalburg, W. (1999). Sentence comprehension and word repetition: A positron emission tomography investigation. Psychophysiology, 36, 786-801.

Stromswold, K., Caplan, D., Alpert, N., \& Rauch, S. (1996). Localization of syntactic comprehension by positron emission tomography. Brain and Language, 52, 452-473.

Vandenberghe, R., Nobre, A. C., \& Price, C. J. (2002). The response of left temporal cortex to sentences. Journal of Cognitive Neursoscience, 14, 550-560.

Vandenberghe, R., Price, C., Wise, R., Josephs, O., \& Frackowiak, R. S. (1996). Functional anatomy of a common semantic system for words and pictures [see comments]. Nature, 383, 254-256.

Wagner, A. D., Koutstaal, W., Maril, A., Schacter, D. L., \& Buckner, R. L. (2000). Task-specific repetition priming in left inferior prefrontal cortex. Cerebral Cortex, 10, 1176-1184. 